Wilfrid Laurier University

Scholars Commons @ Laurier

Physics and Computer Science Faculty

Publications

Physics and Computer Science

1984

\title{
Chaotic Behavior in Coupled Superconducting Weak Links
}

M.A.H. Nerenberg

University of Western Ontario

James A. Blackburn

Wilfrid Laurier University, jabjabjab@cogeco.ca

S. Vik

Wilfrid Laurier University

Follow this and additional works at: https://scholars.wlu.ca/phys_faculty

\section{Recommended Citation}

Nerenberg, M.A.H.; Blackburn, James A.; and Vik, S., "Chaotic Behavior in Coupled Superconducting Weak Links" (1984). Physics and Computer Science Faculty Publications. 29.

https://scholars.wlu.ca/phys_faculty/29

This Article is brought to you for free and open access by the Physics and Computer Science at Scholars Commons @ Laurier. It has been accepted for inclusion in Physics and Computer Science Faculty Publications by an authorized administrator of Scholars Commons @ Laurier. For more information, please contact scholarscommons@wlu.ca. 


\title{
Chaotic behavior in coupled superconducting weak links
}

\author{
M. A. H. Nerenberg \\ Department of Applied Mathematics, Univeristy of Western Ontario, London, Ontario, Canada N6A $5 B 9$
}

James A. Blackburn and S. Vik

Department of Physics, Wilfrid Laurier University, Waterloo, Ontario, Canada N2L 3C5

(Received 25 June 1984)

\begin{abstract}
Computer simulations have been carried out for a system consisting of a pair of coupled superconducting weak links described by a noncapacitive, resistively shunted equivalent circuit. Both dc and ac bias currents are assumed for each link. It is found that for certain ranges of ac amplitude, chaotic behavior occurs. Coupling is crucial to this result-without it no chaos will appear.
\end{abstract}

\section{INTRODUCTION}

The possibility of chaotic behavior in rf-driven Josephson junctions was first suggested by Huberman et al. ${ }^{1}$ in 1980. In the ensuing years a large body of work has appeared on this and related topics. Some representative examples are papers by Kautz, ${ }^{2,3}$ Yeh and Kao, ${ }^{4}$ Ben-Jacob et al., ${ }^{5}$ and Pedersen and Davidson. ${ }^{6}$ In these publications the Josephson element was described by the StewartMcCumber resistively shunted junction (RSJ) model, $^{7}$ with a finite junction capacitance. The resulting secondorder nonlinear differential equations were usually solved by numerical methods, although Cirillo and Pedersen ${ }^{8}$ employed a phase-locked loop circuit as a simulation. Marcus et $a l .{ }^{9}$ investigated the transition to chaos in a voltage-driven resonant junction which included a series inductance. Chaos in of superconducting quantum interference devices (SQUID's) has been studied by Ritala and Salomaa, ${ }^{10}$ and by Kesser et al. ${ }^{11}$ Single- and doublejunction interferometers were examined by Kornev and Semenov ${ }^{12}$ using analog simulators. Again a finite junction capacitance was assumed.

It might be mentioned that experimental evidence for such chaotic behavior is still rather sparse. Miracky, Clarke, and $\mathrm{Koch}^{\mathbf{1 3}}$ reported elevated noise temperatures, attributed to the onset of chaos, in Josephson junctions. Gubankov et al. ${ }^{14}$ have also claimed to see manifestations of chaos in $\mathrm{rf}$-driven superconducting tunnel junctions.

It is well known that superconducting weak links are governed by the Josephson relation $\dot{\phi}=2 \mathrm{eV} / \hbar$, although the current phase relationship might not be exactly sinusoidal. These devices are modeled by a resistivity shunted model without capacitance. Consequently they should not display chaotic behavior in the circuits mentioned above, even when forced by an external ac current, since only one first-order equation, for which a twodimensional phase space applied, arises. ${ }^{15}$ However, when combined as interacting pairs they do show interesting locking phenomena as discussed by Nerenberg et al., ${ }^{16} \mathrm{Dai}$ and Kao, ${ }^{17}$ Neumann et al., ${ }^{18}$ Jillie et al., ${ }^{19}$ and many others. Various coupling mechanisms have been suggested including resistive, resistive-inductive, and charge imbalance. $^{20}$ An interacting pair of weak links is governed by two coupled first-order differential equations. With an external ac basis this system would be represented by a three-dimensional phase space; this suggested the possibility of chaotic behavior. Such a coupled system, together with a single ac-driven capacitive junction, represents the mathematically simplest superconducting circuit in which chaos may occur. The former has the additional feature of chaotic behavior depending crucially, as mutual voltage locking does, on the coupling between weak links. In the absence of coupling, the system is equivalent (except for the synchrony of the external ac currents) to two independent two-dimensional phase spaces in which chaos cannot occur $^{15}$. The conjecture that the coupled pair will display chaotic behavior is the subject of the present work.

\section{EQUATIONS AND MODEL}

The system under consideration consists of two superconducting weak links coupled by an external shunt resistance. $^{21}$ The equivalent circuit shown in Fig. 1 utilizes the noncapacitive RSJ model for the weak links and includes individual bias currents-both dc $\left(I_{1}\right.$ and $\left.I_{2}\right)$ and ac $\left(a_{1} \sin \omega t\right.$ and $\left.a_{2} \sin \omega t\right)$.

In order to express the final equations which govern the circuit in terms of dimensionless time, current, and voltage, we now define certain useful parameters. The critical currents of the weak links are $i_{c 1}$ and $i_{c 2}$; their average is

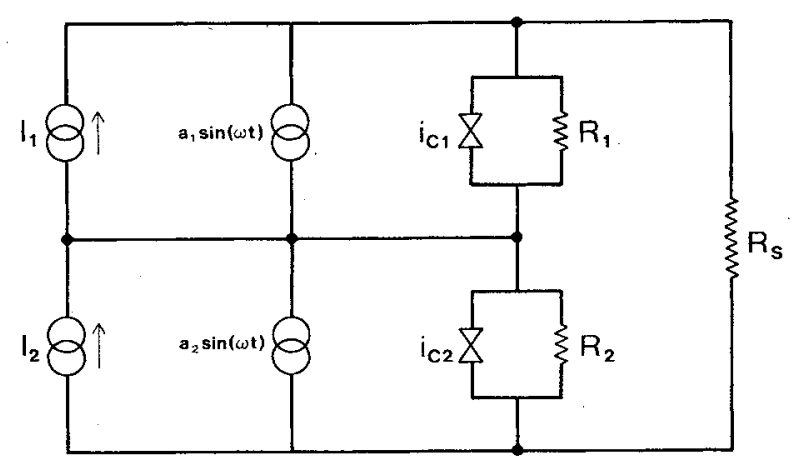

FIG. 1. Schematic of coupled superconducting weak links with ac and dc bias currents. 
denoted $\bar{i}_{c}$. Then

$$
V_{0} \equiv \frac{\bar{i}_{c} R_{1}\left(R_{2}+R_{s}\right)}{\left(R_{1}+R_{2}+R_{s}\right)}
$$

will serve as a unit of voltage. In what follows time will be normalized by $\left(2 e V_{0} / \hbar\right)$ and the units of current will be $\bar{i}_{c}$. Furthermore, we define

$$
\alpha \equiv\left(1+R_{s} / R_{2}\right)^{-1}
$$

and

$$
\delta \equiv \frac{\left(1+R_{s} / R_{1}\right)}{\left(1+R_{s} / R_{2}\right)} .
$$

With these definitions, the circuit is described by the following equations in the phase variables $\phi_{1}$ and $\phi_{2}$ :

$\dot{\phi}_{1}=I_{1}-I_{c 1} \sin \left(\phi_{1}\right)-\alpha\left[I_{2}-I_{c 2} \sin \left(\phi_{2}\right)\right]+b_{1} \sin (\omega t)$,

$\dot{\phi}_{2}=\delta\left[I_{2}-I_{c 2} \sin \left(\phi_{2}\right)\right]-\alpha\left[I_{1}-I_{c 1} \sin \left(\phi_{1}\right)\right]+b_{2} \sin (\omega t)$.

Here we have assumed the series-aiding case referred to in Ref. 16. Dots indicate derivatives with respect to normalized time. In Eqs. (1) and (2), $b_{1}=\left(a_{1}-\alpha a_{2}\right) / \overline{i_{c}}$ and $b_{2}=\left(\delta a_{2}-\alpha a_{1}\right) / \overline{i_{c}}$.

\section{SIMULATION AND TESTS FOR CHAOS}

Before proceeding with a discussion of observed behavior, a few preliminary remarks are in order concerning the numerical solution of the coupled pair of differential equations (1) and (2).

A suitable time step $\Delta t$ is selected. The computer program will print or otherwise output results at these intervals. At the beginning of each new increment in time, $t$, $\phi_{1}, \phi_{2}, \dot{\phi}_{1}$, and $\dot{\phi}_{2}$ are all available from the previous iteration. The computation from $t$ to $t+\Delta t$ is done by sequentially stepping along a subdivision of the interval $(t, t+\Delta t)$ with the aid of an Adams-Moulton predictorcorrector algorithm. This procedure is not self-starting and so the first of the iterations is performed by means of a fourth-order Runge-Kutta formula. Because of the anticipated chaotic regimes, it was apparent that particular care had to be taken with convergence criteria. At various points in the computer runs, cross checks were made to verify consistency with respect to altered computational grids using double precision arithmetic. That such is not a minor concern can be appreciated from the work of Yamaguti and Ushiki, ${ }^{22}$ in which it was demonstrated that the Euler and central difference schemes can produce "ghost solutions" which eventually become chaotic, and that the numerical precision of the calculations can be very important in this regard. However, no indications of this type of behavior were noted in the present work.

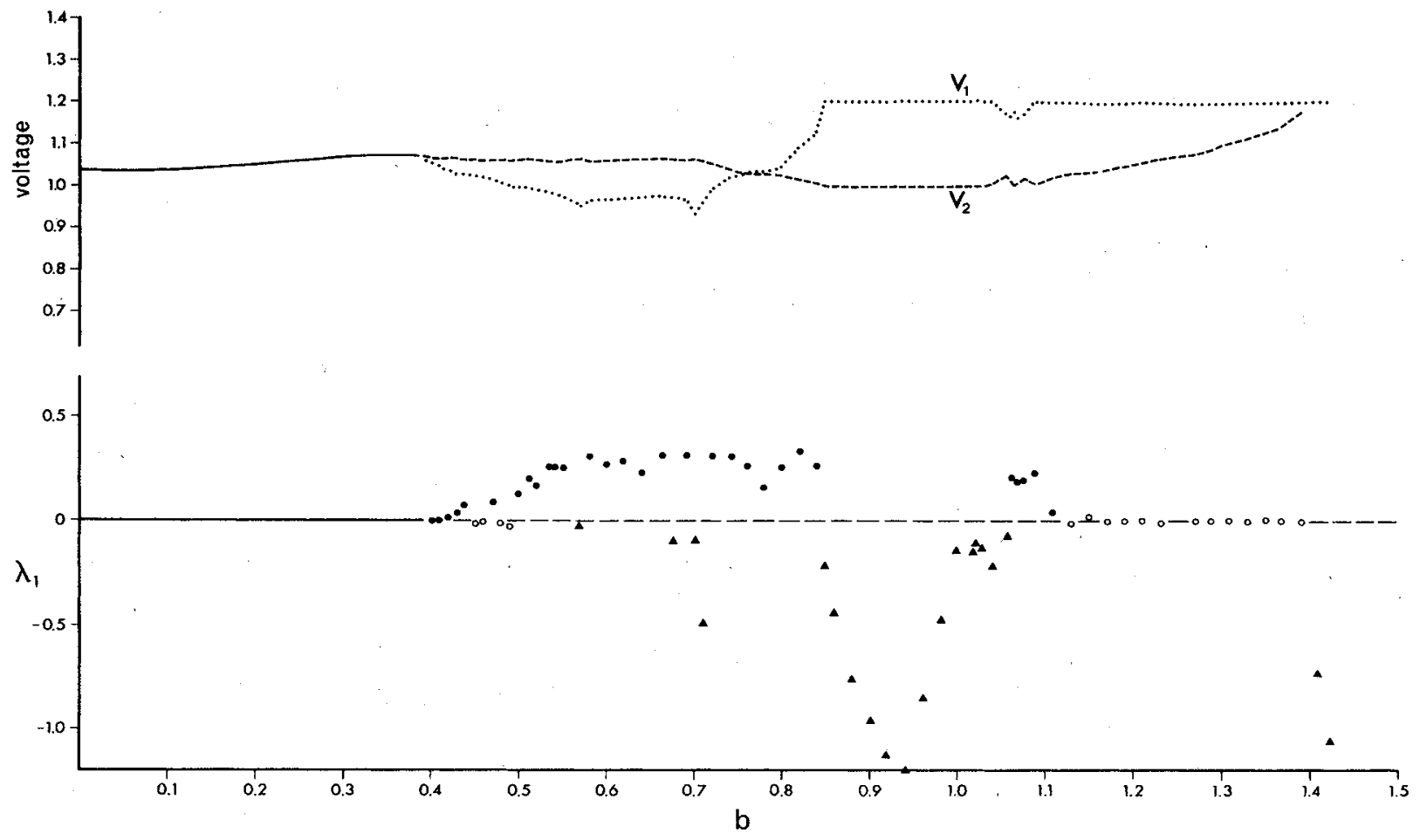

FIG. 2. Upper: dc link voltages as a function of ac drive amplitude. Parameter values are given in the text. Notice that the links remain locked until $b \geq 0.37$. Lower: larger Liapunov number $\left(\lambda_{1}\right)$ versus ac drive amplitude $(b)$. Negative values, shown as triangles, indicate a periodic attractor; positive values, shown as solid circles, correspond to chaotic solutions. For the remaining points (open circles) $\lambda_{1}=0$ within computational precision and the behavior is multiply periodic. 
Next we consider the interpretation of the simulation output, namely $\dot{\phi}_{1}(t)$ and $\dot{\phi}_{2}(t)$. These are just normalized versions of the time-dependent link voltages. It is not always an altogether simple matter to judge from the appearance of these two voltages whether or not the system is in a chaotic state. We therefore chose to perform several independent tests, as follows.

The power spectrum, which involves discrete Fourier analysis, could be evaluated provided a sufficiently long temporal record had been accumulated and provided that aliasing of the spectrum was handled properly. Chaos is expected to manifest itself as a broadband noise base in the spectrum.

A second test for chaos utilized the Liapunov exponents. These can be calculated as the computation proceeds-in contrast to Fourier analysis which requires a finished run. These exponents measure the contraction or expansion of a volume element in phase space. Chaotic solutions are characterized by having a larger exponent $\left(\lambda_{1}\right)$ greater than zero, and smaller exponent $\left(\lambda_{2}\right)$ less than zero. The former is a measure of the exponential divergence of neighboring trajectories which define the "strange" attractor or chaotic solution. Since in dissipative systems there is an overall shrinking of the volume element with increasing time, $\lambda_{2}$ must be negative. Periodic and multiply periodic solutions have $\lambda_{1} \leq 0$ and $\lambda_{2}<0$. A negative larger exponent would indicate that the periodic solution is an attractor towards which neighboring solutions converge with time. That is, such a solution behaves in the opposite way to the strange attractor, in being insensitive to initial conditions.

The procedure for evaluating $\lambda_{1}$ and $\lambda_{2}$ was as follows. Let us say we have arrived at time $t$. Using the (now) available values of $\phi_{1}(t)$ and $\phi_{2}(t)$, one may proceed, as described earlier, to a new time $t+\tau$. Any system which is potentially chaotic will display an extreme sensitivity to initial conditions, and so small changes forced on $\phi_{1}(t)$ and $\phi_{2}(t)$ would lead to wildly different results at $t+\tau$. In other words, one can test the sensitivity of the system by examining the mapping from the neighborhood around $\left(\phi_{1}(t) \phi_{2}(t)\right)$ to the destination at $\left(\phi_{1}(t+\tau), \phi_{2}(t+\tau)\right)$. $\mathrm{Ott}^{23}$ has shown how to extract the two Liapunov coefficients $\lambda_{1}$ and $\lambda_{2}$ by means of such a sensitivity test. Specifically, the results at $t+\tau$ must be computed for three possible initial conditions at $t$, namely $\left(\phi_{1}, \phi_{2}\right)$, $\left(\phi_{1}+\epsilon, \phi_{2}\right)$, and $\left(\phi_{1}, \phi_{2}+\delta\right)$. The three answers at $t+\tau$ are then used in a procedure which yields $\left(\lambda_{1}, \lambda_{2}\right)$. We also used another method due to Benettin et al. ${ }^{24}$ which independently yielded the larger of $\left(\lambda_{1}, \lambda_{2}\right)$. The two techniques gave values in good agreement with each other.

\section{COMPUTATIONAL RESULTS}

Because of the number of variables in Eqs. (1) and (2), it was not practical to attempt to completely map the dynamical states of the system under consideration. Instead, we elected to fix all but two of the parameters in such a way that the coupled pair are locked in the absence of external excitation. Such behavior was already known to us from a previous study (16). Hence we chose $I_{c 1}=1.2, I_{c 2}=0.8, I_{1}=2.0, I_{2}=2.2, \alpha=0.2$, and $\delta=\frac{2}{3}$.
This results in voltage locking with both weak links undergoing periodic phase slip at a frequency $\omega_{0}=1.04$. The choice was made for convenience to conform with the example represented in Fig. 2 of Ref. 16. As chaos may result only after a sequence of bifurcations to increasingly complex behavior, we selected the locked situation as representing the simplest point of departure. Also for convenience in the calculations, we set $b=b_{1}=b_{2}$. This choice is valid provided the original ac source amplitudes
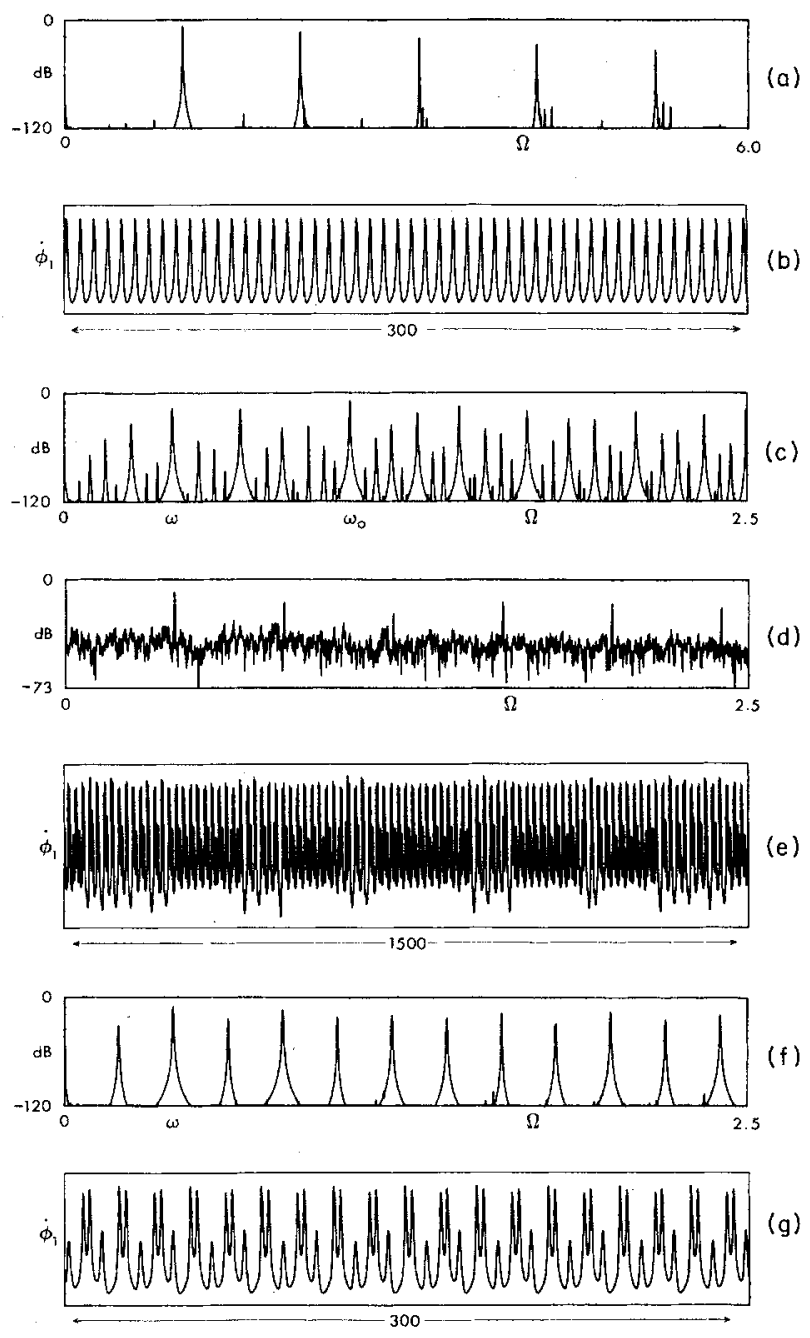

FIG. 3. (a) Power spectrum of link 1 in the absence of an ac bias. Notice that the only frequencies which appear are at $\Omega=\omega_{0} \simeq 1.04$ and its harmonics. (b) Portion of the $\dot{\phi}_{1}$ versus $t$ data corresponding to the situation represented in (a). Periodic behavior is clearly evident. (c) Power spectrum of link 1 with ac bias current $(b=0.2)$. Note that the spectrum now contains peaks at frequencies $\Omega=\omega_{0}, \omega$, and their sums and differences. (d) Same as (a) and (c), but with $b=0.84$. Chaotic behavior is apparent. (e) Same as (b) but with $b=0.84$. (f) Power spectrum for $b=0.86$. Notice the abrupt change compared to the spectrum in (d) for which $b=0.84$. Referring to Fig. 2 it can be seen that the behavior is periodic with $\lambda_{1}<0$. (g) Portion of $\dot{\phi}_{1}$ versus $t$ when $b=0.86$, as in (f). 
are in the ratio $(\delta+\alpha) /(1+\alpha)$. The impressed "rf" field was then assigned a frequency $\omega=0.4$ and the amplitude $b$ was progressively increased in a series of computer runs. Care was taken to wait for transient effects to dissipate (approximately $2000 \mathrm{rf}$ cycles) before storing and analyzing the computational data. The principal features observed may be summarized as follows (refer to Fig. 2).

(a) $0<b<0.37$. Immediately on allowing the external ac to be nonzero, the system goes from periodic behavior to multiply periodic behavior containing the mutual locking frequency $\omega_{0}$ which drifts slowly up in value with increasing $b$, the external frequency $\omega$, and their sums and differences [see also Fig. 3(c)]. These are not chaotic motions-as indicated by the larger Liapunov number being zero throughout.

(b) $0.37<b \leq 0.42$. Here is a small region in which the self-locking of the two weak links is finally broken, but the behavior is still multiply periodic, although it is more complex than in region (a).

(c) $0.42<b \leq 0.84$. In this range of applied voltage the dynamics change, with the larger Liapunov coefficient abruptly going to values greater than zero, indicating chaotic behavior. This is confirmed by the presence of broadband noise in the Fourier spectrum [see also Fig. 3(d)]. Note, however, that this regime is interspersed with narrow intervals where the larger Liapunov coefficient drops to zero or goes negative.

(d) $0.84<b<1.06$. At an applied if current of $b \simeq 0.84$ the chaotic behavior terminates quite abruptly. The larger Liapunov coefficient goes negative indicating a strong periodic attractor. The periodic motion of the links is determined by the external drive frequency. In fact it is observed that throughout this range the two links maintain constant time-averaged voltages which are simply related to the drive frequency. Refer also to Fig. 3(f).

(e) $b>1.06$. At larger applied currents the system first reenters the chaotic domain, then leaves it. This alternating amongst chaos, periodicity, and multiple periodicity might be expected to continue as the external excitation grows stronger.

A further illustration of chaotic behavior is provided by the Poincaré plot shown in Fig. 4. Periodic motion would manifest itself as one or a few fixed points, In contrast this figure is typical of a strange attractor.

\section{DISCUSSION}

The above description of the system is admittedly incomplete. It is possible that more structure is present between data points in Fig. 2. However, certain essential

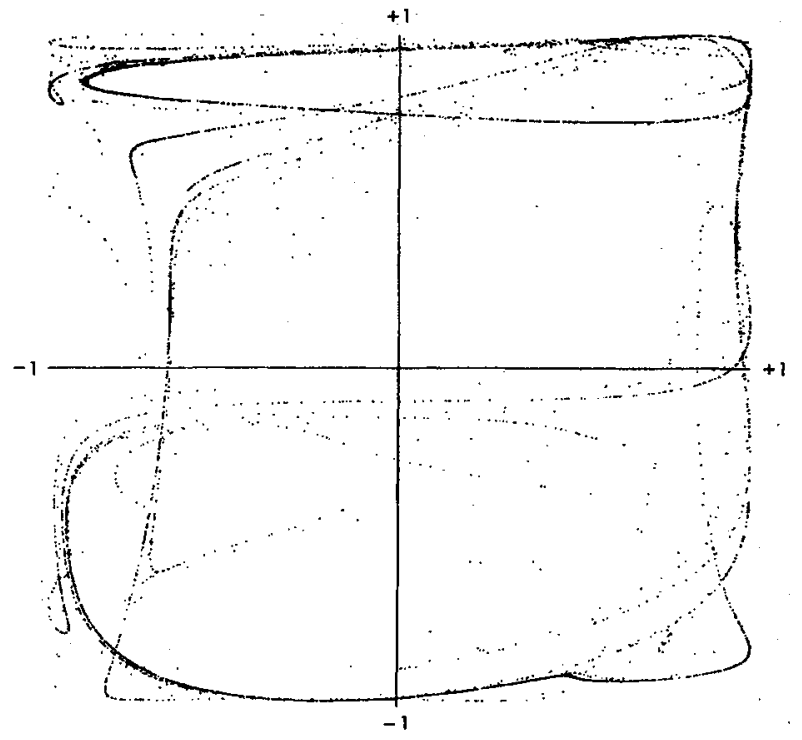

FIG. 4. Poincaré section for $b=0.84$. The figure results from plotting $\sin \phi_{2}$ (vertical) versus $\sin \phi_{1}$ (horizontal) at successive intervals in time separated by $2 \pi / \omega ; 6366 \mathrm{rf}$ cycles have been evaluated.

features stand out. In the first place it is demonstrated that a pair of coupled, noncapacitive superconducting weak links will undergo a transition to chaotic behavior from an initially locked state under the influence of a sufficiently strong ac excitation. Secondly, it has been shown that chaos disappears and reappears as the excitation is progressively made more intense. Finally, we have observed that the route to chaos for this system is not of the period doubling type, but rather more abrupt, sometimes involving complex spectra (e.g., period 11, 22, ...) appearing at the edges of the chaotic domains.

\section{ACKNOWLEDGMENTS}

We are grateful to Dr. H. J. T. Smith, Dr. J. Bindslev Hansen, and Dr. R. L. Kautz for useful discussions relating to chaotic behavior in weak links. One of the authors (M. A. H. N.) would like to thank Dr. Y. Imry for hospitality extended to him during a visit to the University of Tel Aviv, when some of the ideas for this study originated. This work was supported by the Natural Sciences and Engineering Research Council of Canada.
${ }^{1}$ B. A. Huberman, J. P. Crutchfield, and N. H. Packard, Phys. Lett. 37, 750 (1980).

${ }^{2}$ R. L. Kautz, IEEE Trans. Magn. MAG-19, 465 (1983).

${ }^{3}$ R. L. Kautz, J. Appl. Phys. 52, 6241 (1981).

${ }^{4}$ W. J. Yeh and Y. H. Kao, Appl. Phys. Lett. 42, 299 (1983).

${ }^{5}$ E. Ben-Jacob, Y. Brainan, R. Shainsky, and Y. Imry, Appl.

Phys. Lett. 38, 822 (1981).
6N. F. Pedersen and A. Davidson, Appl. Phys. Lett. 39, 830 (1981).

${ }^{7}$ W. C. Stewart, Appl. Phys. Lett. 12, 277 (1968); D. E. McCumber, J. Appl. Phys. 39, 3113 (1968).

${ }^{8} \mathrm{M}$. Cirillo and N. F. Pedersen, Phys. Lett. 90A, 150 (1982).

${ }^{9}$ P. M. Marcus and Y. Imry, Solid State Commun. 41, 161 (1982). 
${ }^{10}$ R. K. Ritala and M. M. Salomaa, J. Phys. C 16, L477 (1983).

${ }^{11}$ K. Fesser, A. R. Bishop, and P. Kumar, Appl. Phys. Lett. 43, 123 (1983).

12 V. K. Kornev and V. K. Semenov, IEEE Trans. Magn. MAG-19, 633 (1983).

${ }^{13}$ R. F. Miracky, J. Clarke, and R. H. Koch, Phys. Rev. Lett. 50, 856 (1983).

${ }^{14}$ V. N. Gubankov, K. I. Konstantinyan, V. P. Koshelets, and G. A. Ovsyannikov, IEEE Trans. Magn. MAG-19, 637 (1983).

${ }^{15}$ O. E. Rossler, in Nonlinear Oscillations in Biology, edited by Frank C. Hoppensteadt (American Mathematical Society, Providence, 1979).

${ }^{16}$ M. A. H. Nerenberg, J. A. Blackburn, and D. W. Jillie, Phys.
Rev. B 21, 118 (1980).

${ }^{17}$ Y. D. Dai and Y. H. Kao, J. Appl. Phys. 52, 4135 (1981).

${ }^{18}$ L. G. Neumann, Y. D. Dai, and Y. H. Kao, Appl. Phys. Lett. 39, 648 (1981).

${ }^{19}$ D. W. Jillie, J. E. Lukens, and Y. H. Kao, Phys. Rev. Lett. 38, 915 (1977).

20J. A. Blackburn, J. Low Temp. Phys. 50, 475 (1983).

${ }^{21}$ Structurally, identical equations arise for other coupling schemes, including quasiparticle diffusion. See Nerenberg et al. (Ref. 16).

${ }^{22}$ M. Yamaguti and S. Ushiki, Physica 3D, 618 (1981).

${ }^{23}$ E. Ott, Rev. Mod. Phys. 53, 655 (1981).

${ }^{24}$ G. Benettin, L. Galgani, and J. M. Strelocyn, Phys. Rev. A 14, 2338 (1976). 